\title{
Coherent quantum states in the laser-induced thin film nanocluster structures: optical and electrophysical properties
}

\author{
S. Arakelian*, A. Kucherik, S. Kutrovskaya, A. Osipov, A. Istratov, and I. Skryabin \\ Vladimir State University named after Alexander and Nikolay Stoletovs, Vladimir, 600000 Russia
}

\begin{abstract}
We studied in both theory and experiment the nanocluster structures of different types (due to topology and composition) taking into account the correlations in nanoparticle-ensemble by quantum states. The problem of high temperature superconductivity due to topological surface structures with localized states has been under our consideration. Dramatic enhancement of electroconductivity (in six orders!), observed in our experiments, may be discussed as a real tendency to high temperature superconductivity due to topological peculiarities of nanocluster system. Near-field photoluminescence spectra/optical response in hybrid nanosystems measured by us demonstrated a big efficiency in the nanoantenna respect.
\end{abstract}

1. A nanocluster system may be presented as a quantum object when it's characteristic size $a \sim \lambda_{\mathrm{dB}}$, where $\lambda_{d B}$ is the de Broglie wavelength. But the principal question is the value of effective mass of electrons in the nanocluster system. For typical semiconductors we can estimate $\lambda_{\mathrm{dB}}=25 \mathrm{~nm}$ being an ordinary nanocluster size. Moreover, for inhomogeneous system the correlated states should be very strong for nanoclusters.

In our experiments we generated some different network nanostructures of both types/dimensions and compositions. Such procedures for both direct laser modification of the solid state surface and laser ablation from target in liquid to produce, first, the colloidal systems and, second, to deposit the nanoparticles from colloid on solid surface are described e.g. in [1] including the hybrid set-up.

In frame of nanocluster network as a Kronig-Penny quantum wells (cf. [1]) we have (in analogy with optics) a local Mie-resonance model for electron wave-functions in contrast with a slowly varying energetic structure being a Bragg-resonance model. In last case we have non-local interference effect, and can speak about correlation of electrons due to existing of spatial structure. We have to separate the two such topology based opportunities: multiple Raman-Nath diffraction (for thin periodic structure) and namely Bragg-diffraction (for bulk structure).

But for electrical conductivity the last case allow us to consider the opportunity to potentially enhance the electroconductivity in some fixed direction. Thus, we can control the directional diagram and indicatrices for the charge particle propagation. Selected directions, i.e. anisotropy, are equivalent to symmetry breaking being a fundamental basis for physical states [3]. In general, two mentioned above cases can be presented as the twophase states on a specific phase-diagram transition between different spatial structures.

* Corresponding author: arak@,vlsu.ru 
2. The electrical transport properties in analogy with the tunnel and quantum correlated states are namely considered; the jump (hopping)/tunneling/thermal activation effects for electroconductivity have been observed in different our experiments (cf. [4,5]).

To analyze the size-depended effect we used a percolation theory by computer simulation procedure [6]. In addition, we take into account the fractal structures of the units, and we estimated the measured value of electroresistance R vs both nanoparticle size (a) and topology by fractal dimension (D) as well.

According to this we had a good fit for the resistivity by theory $\left(R_{\text {cal }}\right)$ and experiment $\left(R_{\text {meas }}\right)$ in a large range of the values (see Fig. 1) for gold $(A u)$ nanostructures as an example. The principal result is a six orders difference (!) in values of $\mathrm{R}$ by topology variation only. The temperature dependences for bi-metallic $(\mathrm{Au}+\mathrm{Ag})$ film are also obtained by us (cf. [7]), and the activation energy for electroconductivity is estimated as $1.3 \mathrm{eV}$.

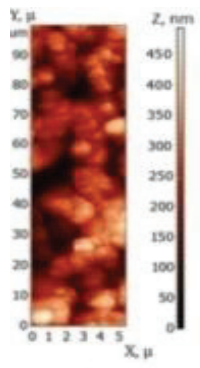

is)

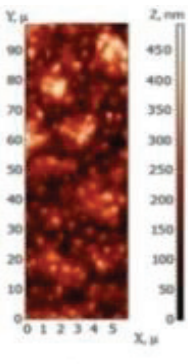

b)

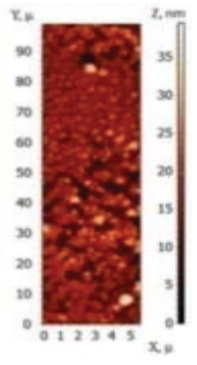

c)

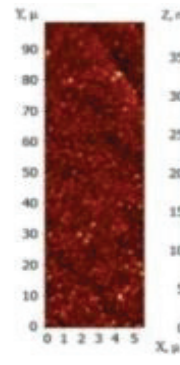

(5)

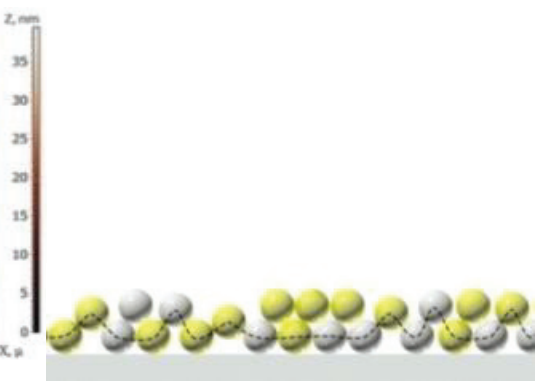

Fig. 1. Experimental and theoretical (by fractal approach) comparison of values for electroresistance $\mathrm{R}$ for Au-nanostructure (AFM-picture): a) $\mathrm{R}_{\text {meas }}=5.4 \cdot 10^{8} \mathrm{Ohm}, \mathrm{D}=1.39, \mathrm{R}_{\text {calc }}=9.3 \cdot 10^{8} \mathrm{Ohm}$; b) $\mathrm{R}_{\text {meas }}=$ $\left.3.6 \cdot 10^{7} \mathrm{Ohm}, \mathrm{D}=1.57, \mathrm{R}_{\text {calc }}=5.5 \cdot 10^{7} \mathrm{Ohm} ; \mathrm{c}\right) \mathrm{R}$ meas $=5.4 \cdot 10^{6} \mathrm{Ohm}, \mathrm{D}=1.84, \mathrm{R}_{\text {calc }}=9.3 \cdot 10^{6} \mathrm{Ohm}$; d) $R_{\text {meas }}=3.6 \cdot 10^{2} \mathrm{Ohm}, \mathrm{D}=1.93$, $\mathrm{R}_{\text {calc }}=5.5 \cdot 10^{2} \mathrm{Ohm}$, where $\mathrm{R}_{\text {meas }}$ - measurements; $\mathrm{R}_{\text {calc }}-$ calculations; D - fractal dimension. The size of the nanoobject: (a),(b) - $100 \mathrm{~nm}$; (c),(d)-10nm. Optimal electron trajectory (in respect of min value of R), i.e. self-organization effect for the two layer system is shown (by dotted curve) in insert on the right.

3. In superconductivity problem (for e-fermions) the phenomenon can be presented as superfluidity (for bosons) when coupled electrons (Cooper-pairs) occur [3]. For cluster system some alternative mechanisms of electron coupling (not via standard phonon coupling) may exist. We discuss for the first time the several new opportunities for that.

4. Future activity in the field should be focused on the more detailed comparison of obtained experimental result for induced (by controllable way) nanocluster topological systems and concrete mechanism of its development under different conditions. The results give us perspective directions for possible applications of the phenomena in hybrid photonic devices (optics + electrophysics) of new generation, especially for optical signals enhancement in respect of nanoantenna (see e.g. [8]).

The work was partially carried out within the framework of RFBR projects 16-42-330461 p-a, 16-42330531 p-a, 17-42-330928 p-a.

\section{References}

[1] S. Arakelian et al., Introduction to the femtonanophotonics (Moscow, Logos, 2015)

[2] V. Gartmakher, Electrons in non-ordered medium (Moscow, Fizmatlit, 2013).

[3] N.Bogolyubov, B.Sadovnikov, Some problems in statistical mechanics (Moscow, 1975)

[4] S. Arakelian et al., Opt. and Quant. Electronics 48(6), 342 (2016)

[5] S. Arakelian et al., J. Nanopart. Res. 18(6), 155 (2016)

[6] S. Arakelian et al., Opt. and Quant. Electronics 48(11), 505 (2016)

[7] A. Kavokin et al., Superlattices and Microstructures 109, 1 (2017)

[8] S. Kutrovskaya S. Arakelian, A. Kucherik et al., Sci. Rep. 7, 10284 (2017) 